Antonio Breno de Alleluia

Geração de Pulsos Ópticos Curtos e Multiplexação no Domínio do Tempo

Dissertação de Mestrado

Dissertação apresentada como requisito parcial para obtenção do título de Mestre pelo Programa de PósGraduação em Engenharia Elétrica da PUC-Rio.

Orientador: Jean Pierre von der Weid 
Antonio Breno de Alleluia

\title{
Geração de Pulsos Ópticos Curtos e Multiplexação no \\ Domínio do Tempo
}

\begin{abstract}
Dissertação apresentada como requisito parcial para obtenção do título de Mestre pelo Programa de PósGraduação em Engenharia Elétrica da PUC-Rio. Aprovada pela Comissão Examinadora abaixo assinada.
\end{abstract}

\author{
Jean Pierre von der Weid \\ Orientador \\ CETUC PUC-RIO \\ Jean Pierre von der Weid \\ CETUC PUC-RIO \\ Alexandre Dal Forno \\ UERJ
}

\section{Luis Carlos Blanco Linares}

MEC PUC-RIO

Rogerio Passy

MLS Wireless

Marbey Mosso

CETUC PUC-RIO

Prof. José Eugenio Leal

Coordenador(a) Setorial do Centro Técnico Científico - PUC-Rio 
Todos os direitos reservados. É proibida a reprodução total ou parcial do trabalho sem autorização da universidade, do autor e do orientador.

\section{Antonio Breno de Alleluia}

Graduou-se em engenharia elétrica com ênfase em telecomunicações na Universidade Estadual do Rio de Janeiro. Iniciou o mestrado com o grupo de optoeletrônica do Centro de Estudos em Telecomunicações da Pontifica Universidade Católica do Rio de Janeiro, desenvolvendo pesquisa na área de fenômenos não lineares ligados a geração e multiplexação óptica de pulsos curtos.

Ficha Catalográfica

Alleluia, Antonio Breno

Geração de pulsos curtos ópticos e multiplexação no domínio do tempo / Antonio Breno de Alleluia ; orientador: Jean Pierre Von der Weid. - 2006.

$88 \mathrm{f.}$;

Dissertação (Mestrado em Engenharia Elétrica)Pontifícia Universidade Católica do Rio de Janeiro, Rio de Janeiro, 2006.

Inclui bibliografia

1. Engenharia elétrica - Teses. 2. Gerador. 3. Multiplexador óptico. 4. Pulsos curtos. 5. Mode locked fiber ring laser. I. Weid, Jean Pierre Von der. II. Pontifícia Universidade Católica do Rio de Janeiro. Departamento de Engenharia Elétrica. III. Título.

CDD: 621.3 
A minha família e meus irmãos e irmãs do Laboratório de Optoeletrônica pelo amor e fé depositados em mim para realização desta tese. 


\section{Agradecimentos}

Minha sincera gratidão ao professor doutor Jean Pierre que me recebeu como o filho pródigo junto ao laboratório de óptica por toda dedicação,amizade e companheirismo no auxílio para minha formação científica. Meus companheiros de laboratório Giancarlo,Claiton,Guilherme e Janaína pela disponibilidade e atenção.

Meu professor Dal Forno o qual me apresentou o mundo da óptica durante o período que fui aluno na Universidade Estadual do Rio de Janeiro.

Ao meu amigo Djeisson Hoffaman que durante todo o período da tese travou diálogos científicos fundamentais para a execução operacional da tese referente as mais diversas questões. Uma declaração a parte para o Claiton que na qualidade de melhor artesão projetou o violão para as linhas de atraso que infelizmente não foram utilizados mas que o conhecimento contribuiu para projetar multiplexador óptico passivo.

Os estagiários Marçal e Junior pela acessória em esquemas eletrônicos requeridos durante o período.

Aos técnicos Nilson,Tonhão e Gutenberg pelo auxílio desenhos envolvidos durante o período.

Minha grande amiga Amália que esteve além de todas as expectativas de secretária mas apresentou-se como uma mãe em muitas situações.

Minha família, a qual sou eternamente devoto por todo o investimento financeiro e emocional empregado para realização desta pesquisa sem a qual o sucesso deste 
trabalho não teria ocorrido.

Minhas vovós que sempre vibravam ao meu lado e queriam entender o que tanto eu fazia trancafiado em um laboratório nos finais de semana.

Uma pessoa amada e querida que durante um grande período acompanhou, sofreu e sorriu com toda a tese e muito a desejou concluída.

Ao CNPQ pelo apoio e financiamento para execução da tese. 


\section{Resumo}

Alleluia,AntonioBreno;von der Weid, Jean Pierre.Geração e Multiplexação Óptica de Pulsos Curtos. Rio de Janeiro, 2006. 88p. Dissertação de Mestrado - Departamento de Engenharia Elétrica, Pontifícia Universidade Católica do Rio de Janeiro.

Esta dissertação de mestrado demonstra o princípio do funcionamento de um sistema gerador e multiplexador óptico utilizando pulsos curtos. Através do efeito de mode locked fiber ring laser pulsos curtos ópticos são gerados para taxa de repetição de $2.48 \mathrm{GHz}$ ao passo que o escalonamento é atribuído ao uso de dispositivos passivos ópticos os quais compõem o conjunto experimental permitindo que a freqüência de $9.95 \mathrm{GHz}$ seja atingida mediante a combinação exata das técnicas utilizadas. O coração de sistema está contido na geração e controle dos pulsos os quais determinarão a taxa de repetição em baixa frequiência e posteriormente a multiplexação através de linhas de atraso devidamente combinadas fornecerão o valor escalonado de operação de frequência.

\section{Palavras-chave}

gerador;multiplexador óptico;pulsos curtos;mode locked fiber ring laser 


\section{Abstract}

Alleluia, Antonio Breno;von der Weid, Jean Pierre . Generation of optical Short Pulses and Time Domain Multiplexing. Rio de Janeiro, 2006. 88p. MSc. Thesis - Departamento de Engenharia Elétrica, Pontifícia Universidade Católica do Rio de Janeiro.

This master thesis demonstrates the principle of the functioning of an optical time domain multiplex system using short pulses. Through the effect of mode locked to fiber ring laser, optical short pulses are generated at repetition of 2.48 $\mathrm{GHz}$ to the step that the scheduling is attributed to the use of optic passive devices which compose the experimental setup allowing that the frequency of $9.95 \mathrm{GHz}$ is reached by means of the accurate combination of the used techniques. The system heart is contained in the generation and control of the pulses which will later determine the rate of repetition in low frequency and multiplexing through lines of delay duly combined.

\section{Keywords}

Generator, multiplex;mode locked fiber ring laser 
"E disse Deus: Haja Luz. E houve luz. Viu Deus que a luz era boa, e fez separação entre a luz e as trevas" 


\section{Sumário}

1 .Introdução

2.Gerador Óptico 18

2.1.1. Dispersão da Velocidade de Grupo 18

2.1.2. Auto Modulação de Fase 19

2.1.3. Equação Não linear de Schrödinger 20

2.1.4. Mode Locked Fiber Ring Laser 20

2.1.5. Análise Computacional para um Sistema de Laser em Anel Simples $\quad 21$

2.1.6. Mode locked experimental 25

2.1.7. Mode Locked Racional $\quad 45$

3 Considerações para estabilização dos modos da cavidade. 57

4.Multiplexação Óptica no Domínio do Tempo 61

4.1.1. Processamento e transmissão de dados no domínio óptico 62

4.1.2. Multiplexação Óptica no Domínio do Tempo 63

4.1.3. Medidas de multiplexação óptica 64

5 Comentários e Conclusões $\quad 75$

$\begin{array}{ll}6 \text { Referências bibliográficas } & 79\end{array}$

7 . Apêndice Alpha 82 


\section{Lista de figuras}

Figura 1 Enlace óptico DWDM 14

Figura 2 Pulsos Multiplexados opticamente no domínio do tempo 15

$\begin{array}{ll}\text { Figura } 3 \text { Oscilador óptico } & 21\end{array}$

Figura 4 Simulação dos pulsos travados $\quad 22$

Figura 5 Pulsos simulados com maior número de modos 23

Figura 6 Travamento dos pulsos simulados 23

Figura 7 Interferência destrutiva resultante do descasamento de fase 24

Figura 10 Setup experimental 26

Figura 11 Tempo dentro da cavidade $\quad 28$

Figura 12 Transformada do sinal na cavidade 29

Figura 13 Sinal travado dentro da cavidade 30

Figura 14 Modulação para sinal de $10 \mathrm{MHz} \quad 31$

Figura 15 Modulação para sinal de $58 \mathrm{MHz} \quad 31$

Figura 16 Transformada de Fourier do Sinal 32

Figura 17 Pulso gerado pelo travamento dos modos 33

Figura 18 FFT do pulso de 112ps 34

Figura 19 Pulsos simulados no Maplev 35

Figura 20 Pulsos gerados pelo travamento dos modos 35

$\begin{array}{ll}\text { Figura } 22 \text { FFT do pulso de } 49.75 \text { ps } & 37\end{array}$

Figura 23 Pulsos gerado pelo travamento dos modos 37

Figura 24 Espectro do Sinal travado no ESA 38

Figura 25 Pulsos simulados $\quad 39$

Figura 26 Pulso de 45.29 ps. 40

Figura 27 FFT do pulso 45.29 ps $\quad 40$

Figura 28 Pulso gerado fora de sintonia $\quad 41$

Figura 29 FFT do pulso $\quad 42$

Figura 30 Pulso gerado fora do harmônico exato. 42

$\begin{array}{ll}\text { Figura } 31 \text { FFT do pulso } 72.04 \text { ps } & 43\end{array}$

Figura 33 FFT pulso 73.22 ps 44

Figura 34 Pulso 22ps 45 
Figura 35 FFT do pulso de 22 ps

Figura 36 Pulso de 28.11 ps

Figura 37 FFT do pulso de 28.11 ps 47

Figura 38 Pulso de 34 ps 48

Figura 39 FFT do pulso de 34ps 49

Figura 40 Pulso de 38.70 ps $\quad 50$

Figura 41 FFT pulso de 38.70ps 51

Figura 42 Pulso 30,54 ps. 51

Figura 43 Pulso de 28.11 ps 52

Figura 44 FFT pulso de 28.11 ps 53

Figura $45 \mathrm{FFt}$ pulso de 30.54ps. $\quad 54$

Figura 46a Pulso de referência $\quad 55$

Figura 47 Setup experimental $\quad 57$

Figura 48 Geladeira do MLFRL $\quad 59$

Figura 49 Sistema OTDM montado em bancada 63

Figura 50 Setup de geração e multiplexação 64

Figura 51 Canal de referência 65

Figura 52 Canais 1 e $2 \quad 66$

Figura 53 Canais superpostos $\quad 67$

Figura 54 Canais 1 e $4 \quad 67$

Figura 55 Canais 2 e $3 \quad 68$

Figura 56 Canais 2 e $4 \quad 69$

Figura 57 Canal 3 e $4 \quad 69$

Figura 59 Canal $2 \quad 71$

Figura 60 Canal $3 \quad 72$

Figura 61 Canais após ajuste de linhas $\quad 72$

Figura 62 Pulsos multiplexados 73 